\title{
Enfermedad vascular cerebral: factores de riesgo y prevención secundaria
}

Villanueva JA. Enfermedad vascular cerebral: factores de riesgo y prevención secundaria. An Med Interna (Madrid) 2004; 21 : $159-160$.

La enfermedad vascular cerebral (EVC) es una de las primeras causas de ingreso hospitalario. Aunque sobreviene a cualquier edad, su incidencia se incrementa a partir de los 55 años, edad en la que se concentra la enfermedad aterosclerótica. Su elevada incidencia (entre 150-350 casos/100.000 habitantes/año, con amplias variaciones) representa la primera causa de invalidez, un riesgo elevado de demencia además de conllevar una elevada mortalidad. En efecto, alrededor de un $10 \%$ de las muertes en países industrializados tienen por causa la EVC, así como el elevado número de infartos silentes demostrados por resonancia magnética cráneo-encefálica, unas cifras que justifican el compromiso de poner en marcha todas las medidas disponibles dedicadas a su prevención y tratamiento (1-3).

Debido a un manejo clínico más eficiente durante la fase aguda y al mejor control de los factores de riesgo, hemos asistido durante las últimas décadas a una importante reducción en la mortalidad de la EVC, posiblemente reducida en un $50 \%$. Ciertas variaciones entre distintos estudios podrían explicarse por diferencias en los criterios diagnósticos aplicados (4). Los estudios epidemiológicos, aparte de mejorar el conocimiento de su historia natural, han demostrado que la identificación de factores de riesgo evitables puede tener un impacto positivo en la prevención. En otras palabras, la tragedia del ictus puede prevenirse. Las medidas de prevención primaria incluyen una dieta sana, abandono de hábitos tóxicos, como tabaco o consumo excesivo de alcohol, así como evitando situaciones de estrés. La detección y tratamiento de la hipertensión arterial, dislipemias, cardiopatías y estenosis de carótida son puntos importantes en la prevención.

La definición de ictus propuesta por la OMS (5) incluye aquellos signos clínicos de déficit focal o global con síntomas que persisten durante 24 o más horas o bien muerte sin otra causa aparente. Se excluyen de ésta definición los ataques isquémicos transitorios (AIT), hematoma subdural, así como hemorragias o infartos causados por infección o tumor. Las variaciones de la incidencia observadas en estudios epidemiológicos de distintas regiones geográficas se explican no solo por diferencias en los hábitos de alimentación sino probablemente por problemas metodológicos en la recogida de datos. En España, datos recogidos en diferentes lugares del país, como Alicante, Asturias,
Gerona, Lugo, Murcia, Sabadell y Valencia muestran variaciones de la incidencia que oscilan entre 50 y 323 casos $/ 100.000$ habitantes/año $(6,7)$. Básicamente coinciden con el estudio publicado en este mismo número de la revista (8).

Es evidente que la incidencia de EVC se incrementa con la edad, especialmente a partir de los 65 años. Se estima que entre pacientes mayores de 75 años que superan un primer episodio de isquemia cerebral, el riesgo de sufrir un segundo ictus alcanza 50-75\%. Estos datos indican la importancia de aplicar medidas estrictas de prevención actuando sobre factores de riesgo que podemos modificar.

La hemorragia cerebral tiene una elevada mortalidad, especialmente durante las 72 horas siguientes a su inicio. La mortalidad por hemorragia subaracnoidea e intracerebral se estima entre un 26 y $29 \%$, respectivamente. La edad media de éstos pacientes es menor que la edad media de los ictus en general (56,6 años y 72,1 años, respectivamente) (9). La muerte durante la fase aguda a menudo depende de complicaciones médicas. Entre éstas se encuentra la enfermedad cardiaca, trombosis venosa profunda, tromboembolismo pulmonar, así como infecciones de las vías respiratorias y tracto urinario. Los ictus dejan secuelas no solo motoras y cognitivas, sino otras que pueden empeorar la calidad de vida de aquellos que sobreviven a la fase aguda. Se incluyen entre estas la depresión (10), propensión a las caídas derivada de los déficit motores (11), espasticidad asociada (12), así como la perdida del control esfinteriano (13).

\section{PREVENCIÓN SECUNDARIA}

La prevención secundaria es aplicable tanto en aquellos pacientes que han sufrido un infarto cerebral como aquellos otros que experimentaron un AIT. En todos ellos el riesgo de una recaída es mayor que en la población general. Johnston y cols. (14) siguieron pacientes diagnosticados en un servicio de urgencias de AIT. En los tres meses siguientes el 10,5\% había sufrido un infarto cerebral, de ellos la mitad en las 48 horas siguientes al episodio.

Gorelick y cols. (15), tras revisar la literatura médica, concluyen que tras un episodio de ictus los hábitos que más influyen 
en una posible recaída son el tabaco, alcohol, la actividad física y la dieta. A su vez, los seis factores de riesgo más significativos fueron hipertensión arterial, historia de infarto de miocardio, fibrilación auricular no valvular, diabetes mellitas, dislipemia y estenosis de carótida asintomática. Podríamos resumir diciendo que el control de todas estas variables son las bases esenciales de la prevención primaria del ictus y sus recaídas.

Destacaríamos la hipertensión arterial como uno de los objetivos primordiales en la prevención de la enfermedad vascular cerebral. Su importancia ha sido demostrada por los estudios HOPE (16) y LIFE (17). En el ensayo Progress (18), se incluyeron 6.105 pacientes aleatorizados a un tratamiento activo con peridompril, un IECA, con o sin un diurético asociado, contra placebo. En el brazo bajo tratamiento activo la reducción del ictus fue del $28 \%$ comparado con placebo, pero también se redujeron la incidencia de infarto de miocardio y hemorragia cerebral. El beneficio alcanzó tanto a pacientes hipertensos como normotensos. El tratamiento con fármacos antihipertensivos reduce la incidencia de ictus tanto en el varón (34\%), como en la mujer (38\%), así como pacientes con edad superior a 80 años (34\%) y jóvenes hipertensos o aquellos que han sufrido un episodio cerebrovascular sea un AIT o un ictus establecido (18).

Podríamos así concluir que la disminución en la incidencia de accidentes cerebro-vasculares está más relacionada con la reducción de la presión arterial que con el tipo de fármaco antihipertensivo utilizado. La medicación antihipertensiva está indicada en pacientes que han sufrido un ictus salvo en aquellos que cursen con hipotensión o que exista una contraindicación formal para este tratamiento (19).

\section{Bibliografía}

1. Khaw K. Epidemiology of stroke. J Neurol Neurosurg Psychiatry 1996; 61: 333-8.

2. Príncipe M, Ferretti C, Casini AR, Santini M, Giubilei F, Culasso F. Stroke, disability, and dementia. Results of a polpulation survey. Stroke 1997; 28: 531-6.

3. Leary MC, Saver JF. Incidence of silent stroke in the United States. Poster presented at the 26th American Heart Association Stroke Meeting, February, 2001.

4. Sandercock P, Molyneaux A, Warlow C. Value of computerised tomography in patients with stroke: the Oxfordshire Community Stroke Project. BMJ 1985; 290: 193-7.

5. Tunstall Pedoe H, for the WHO Monica Project Principal Investigators The WorldHealth Organisation. Monica Project. J Clin Epidemiol 1988; 41: 105-14.

6. López-Pousa S, Vilalta J, Llinás J. Incidencia de la enfermedad vascular cerebral en España: estudio en un área rural de Girona. Rev Neurol (Barc) 1995: 23: 1074-80.

7. Caicoya M, Rodríguez T Lasheras C, et al. Stroke Incidence in Asturias, Spain 1990-1991. Rev Neurol 1996; 24: 806-811.

8. Sanclemente Ansó C, Alonso Valdés F, Rovira Pujol E, Vigil Martin D, Vilaró Pujals J. Accidentes vasculares cerebrales en la comarca de Osona. Factores de riesgo cardiovascular. An Med Interna (Madrid) 2004; 21: 161-165.

9. Williams GR, Jiang JG, Matchar DB, Samsa GP. Incidence and occurrence of total (first-ever and recurrent) stroke. Stroke 1999; 30: 25232528.

10. Whyte EM, Mulsant BH. Post stroke depression: epidemiology, pathophysiology, and biological treatment. Biol Psychiatry 2002; 52: 253 264

11. Teasell R, McRae M, Foley N, Bhardwaj A. The incidence and consequences of falls in stroke patients during inpatient rehabilitation: factors associated with high risk. Arch Phys Med Rehabil 2002; 83: 329-333
Se ha discutido si la utilización de estatinas está justificada en la prevención de la EVC. Su utilización no solo busca una reducción de las cifras de lípidos plasmáticos. Ejerce también efectos positivos estabilizando la placa ateroesclerótica, reduce los radicales libres así como los marcadores de la inflamación, además de poseer un efecto antiplaquetario $(20,21)$. Con todo, su indicación en pacientes que han experimentado un infarto cerebral o un AIT no está en estos momentos bien establecida, a diferencia de pacientes con enfermedad coronaria o vascular periférica. Es de esperar que un estudio, en estos momentos en marcha, el SPARCL (22), probablemente ayudara a esclarecer si los pacientes cerebrovasculares podrían beneficiarse de las estatinas como medida de prevención secundaria.

En suma, existe hoy suficiente evidencia a favor de un tratamiento agresivo en el control de los factores de riesgo, una actitud puede reducir significativamente nuevas recaídas tras un episodio de isquemia vascular (16-18). Es notable que, conociéndose sus beneficios, la conducta médica no sea ni uniforme ni suficientemente agresiva, un extremo que subrayan los autores del artículo publicado en este número (8). Por último, es evidente que la terapia antiagregante o medicación anticoagulante representan medidas eficaces en la prevención de recaídas de la enfermedad cerebrovascular en aquellos pacientes que esté indicado.

\section{J. A. VILLANUEVA}

\section{Servicio de Neurología. Hospital General Universitario Gregorio Marañón. Madrid}

12. Watkins CL, Leathley MJ, Gregson JM, Moore AP, Smith TL, Sharma AK. Prevalence of spasticity post stroke. Clin Rehabil 2002; 16: 515522.

13. Brittain K. Stroke and continence care. Nurs Times 2001; 97: 57.

14. Johnston SC, Gress DR, Browner WS, Sidney S. Short-term prognosis after emergency department diagnosis of TIA. JAMA 2000; 284: 29016.

15. Gorelick PB, Sacco RL, Smith DB, Alberts M, Mustone-Alexander L, Rader D, et al. Prevention of a first stroke. A review of guidelines and a multidisciplinary consensus statement from the National Stroke Association. JAMA 1999; 281: 1112-1120.

16. Yusuf S, Sleight P, Pogue J, Bosch J, Davies R, Dagenais G. Effects of an angiotensin- converting-enzyme inhibitor, ramipril, on cardiovascular events in high-risk patients: The Heart Outcomes Prevention Evaluation Study Investigators. N Engl J Med 2000; 342: 145-153.

17. Dahlof B, Devereux RB, Kjeldsen SE, Julius S, Beevers G, Faire U, et al. Cardiovascular morbidity and mortality in the Losartan Intervention for End point reduction in hypertension (LIFE): A randomized trial against atenolol. Lancet 2002; 359: 995-1003.

18. Progress Collaborative Group. Randomized trial of a perindopril-based blood-pressure-lowering regimen among 6105 individuals with previous stroke or transient ischemic attack. Lancet 2001; 358: 1033-1041.

19. Aronow WS, Frishman WH. Treatment of hypertension and prevention of ischemic stroke. Curr Cardiol Rep 2004; 6 (2): 124-9.

20. Rosenson RS, Tangney CC. Antiatherothrombotic properties of statins. Implications for cardiovascular event reduction. JAMA 1998; 279: 1643-1650.

21. Vaughan CJ, Delanty N. Neuroprotective properties of statins in cerebral ischemia and stroke. Stroke 1999; 30: 1969-1973.

22. Callahan A. Cerebrovascular disease and statins: A potential addition to the therapeutic armamentarium for stroke prevention. Am J Cardiol 2001; 88: 33J-37J. 